\title{
First order irrationality criteria for series
}

\author{
LEE A. BUTLER
}

\begin{abstract}
Following a question of Brun, we consider the possibility of finding a first order statement that the rational summands in an infinite series can be checked against in order to give a sufficient condition for the series to sum to an irrational value. We show that such a statement can exist in structures that define the integers, but not in o-minimal structures.
\end{abstract}

2010 Mathematics Subject Classification 11J72 (primary); 03C64 (secondary)

Keywords: irrationality criteria

\section{Introduction}

A notoriously difficult problem in mathematics is proving that a given real number is irrational.

Some numbers enjoy special properties which lend themselves to establishing irrationality. The elegant continued fraction for $e$, for example, immediately implies its irrationality. The irrationality of square roots of non-square integers follows readily from the unique factorisation of the integers. In most cases, though, a proof of irrationality works by appealing to some irrationality criterion. In general these require one to have a sequence of rational numbers that converges to the real number under consideration. The criterion will then say: "If this sequence of rational numbers has a certain property then its limit is irrational."

The criterion of choice for irrationality proofs is the one based upon Dirichlet's approximation theorem. A less popular criterion due to its rarely met hypotheses is one developed by Brun [2] in 1910. It goes as follows.

Theorem 1.1 Let $\left(x_{n}\right)$ and $\left(y_{n}\right)$ be two sequences of positive integers and $L$ be a positive real number such that the following properties hold for all $n \geq 1$ :

(1) $x_{n+1}>x_{n}$ 
(2) $y_{n+1}>y_{n}$

(3) $\frac{x_{n+1}}{y_{n+1}}>\frac{x_{n}}{y_{n}}$

(4) $\frac{x_{n+2}-x_{n+1}}{y_{n+2}-y_{n+1}}<\frac{x_{n+1}-x_{n}}{y_{n+1}-y_{n}}$

(5) $\lim _{n \rightarrow \infty} \frac{x_{n}}{y_{n}}=L$.

Then $L$ is irrational.

By considering the sequence of partial sums, most irrationality criteria such as Brun's can be adapted to test some property of the rational summands in an infinite series rather than terms in a sequence (cf Theorem 2.1). Another thing that irrationality criteria have in common is that they split up the rational numbers in question, considering the numerators and denominators in markedly different roles. This is perhaps not surprising since all known irrationality criteria stem from the same basic geometric observation: if $\alpha$ is irrational then there are points in $\mathbb{Z}^{2}$ arbitrarily close to the line $y=\alpha x$. Applying this observation means finding rational numbers close to $\alpha$, where "close" is dependent only on the size of the denominator.

Surprising or not, Viggo Brun asked in a second paper whether this asymmetry in the treatment of numerators and denominators was really necessary (Brun and Knudsen [3]). He considered real numbers given by infinite sums of rational numbers, say

$$
L=\sum_{n=1}^{\infty} a_{n}
$$

where $a_{n} \in \mathbb{Q}$ for all $n$. He then asked if there was some simple combination of inequalities and limits in functions of finitely many consecutive summands that would detect irrationality - things like

$$
\lim _{n \rightarrow \infty} \frac{a_{n+1}}{a_{n}}=0
$$

which would rule out geometric series, or

$$
\frac{1}{a_{n+1}}>\frac{1}{a_{n}^{2}}-\frac{1}{a_{n}}+1
$$

which will look promising after Corollary 2.2. For every putative irrationality criterion of this form Brun's co-author Finn Faye Knudsen was able to demonstrate a rational sequence $\left(a_{n}\right)$ that satisfied all the criteria but summed to a rational value. Worse, he showed that any criterion of this form that used inequalities and limits in finitely many 
continuous functions would be satisfiable by some rational sequence with a rational sum.

This rather knocks on the head the notion of an irrationality criterion that doesn't "see" the denominators in its input. But an irrationality criterion needn't necessarily be a list of inequalities and limits involving continuous functions. In this paper we consider the possibility of irrationality criteria of the kind that Brun wanted but that are first order statements in some structure with domain contained in $\mathbb{R}$. We will show that the existence of such criteria is linked to the tameness of the structure in question.

In the next section we will first derive an irrationality criterion for series using Brun's criterion above. Then we will see that in some structures we can get an irrationality criterion that takes the summands whole, not as numerators and denominators separately. However, it will be clear that this is a bit of flimflammery since the criterion will either inspect or control the numerators and the denominators in the summands. This may be because some special infinite subset of $\mathbb{Q}$ is definable, allowing one to check whether the summands belong to this set. Less directly, if the integers are definable and, for example, so is the ordering relation, then there is a definable function that extracts the numerator and denominator of a rational number and this function can be called upon within the proposed irrationality criterion. In both these cases the problem is the presence of infinite, discrete definable sets.

In the final section we will consider a class of structures where this kind of trickery can't take place, namely o-minimal structures expanding the real field. Here we will see that any putative irrationality criteria will either reduce to the kind of thing that Knudsen showed to be impossible or else be so restrictive that it becomes a question on the exceptional set of definable functions rather than any general criterion.

Throughout this paper the (strictly) positive rational numbers will be denoted $\mathbb{Q}^{+}$and similarly $\mathbb{R}^{+}$will be the positive reals. Recall that given some subset $X$ of the real numbers the set $\ell^{1}(X)$ is the set of sequences all of whose terms are in $X$ and whose series are absolutely convergent. The term "eventually" with respect to sequences $\left(a_{n}\right)$ will be shorthand for "there exists $N \in \mathbb{N}$ such that for all $n>N \ldots$... Definable will always mean definable with parameters.

\section{Some irrationality criteria for series}

While Brun's criterion ostensibly works only on numbers that are given as the limit of some sequence of rational numbers, it can be used to find a criterion for sums too, albeit one even harder to apply than the original. 
Theorem 2.1 Let $\left(p_{n}\right)$ and $\left(q_{n}\right)$ be two sequences of positive integers with $q_{n}>1$ for all $n>1$ and such that the series $\sum_{n=1}^{\infty} p_{n} / q_{n}$ is convergent, say to some real number L. Suppose that for all $n>1$

$$
\frac{p_{n}}{q_{n}\left(q_{n}-1\right)}>\frac{p_{n+1}}{q_{n+1}-1}
$$

Then $L$ is irrational.

Proof We will take $x_{n} / y_{n}$ to be the $n$th partial sum of the series, and then see what conditions need to be imposed on $p_{n}$ and $q_{n}$ before Brun's criterion can be applied. So let

$$
\frac{x_{n}}{y_{n}}=\sum_{k=1}^{n} \frac{p_{k}}{q_{k}} .
$$

Note that Brun's criterion doesn't require $\operatorname{hcf}\left(x_{n}, y_{n}\right)=1$ so there is no need to worry about this possibility. Since we're not cancelling common terms we can explicitly write down the recursion formulae

$$
\begin{aligned}
& y_{n+1}=q_{n+1} y_{n} \\
& x_{n+1}=q_{n+1} x_{n}+p_{n+1} y_{n}
\end{aligned}
$$

with $x_{1}=p_{1}$ and $y_{1}=q_{1}$. Clearly the first three conditions in Brun's criterion are met by $x_{n}$ and $y_{n}$, and so it remains to check his fourth condition, namely that

$$
\frac{x_{n+2}-x_{n+1}}{y_{n+2}-y_{n+1}}<\frac{x_{n+1}-x_{n}}{y_{n+1}-y_{n}} .
$$

Using the recursion formulae one can check that

$$
\frac{x_{n+1}-x_{n}}{y_{n+1}-y_{n}}=\frac{x_{n}}{y_{n}}+\frac{p_{n+1}}{q_{n+1}-1}
$$

and so the required inequality is equivalent to

$$
\frac{x_{n+1}}{y_{n+1}}+\frac{p_{n+2}}{q_{n+2}-1}<\frac{x_{n}}{y_{n}}+\frac{p_{n+1}}{q_{n+1}-1} .
$$

Since

$$
\frac{x_{n+1}}{y_{n+1}}-\frac{x_{n}}{y_{n}}=\frac{p_{n+1}}{q_{n+1}}
$$

the desired inequality is the same as

$$
\frac{p_{n+2}}{q_{n+2}-1}<\frac{p_{n+1}}{q_{n+1}-1}-\frac{p_{n+1}}{q_{n+1}}
$$

which is the claimed condition. 
In the simplest case, when $p_{n}$ is identically 1 , this criterion says the following.

Corollary 2.2 If $q_{n}$ is a sequence of positive integers with $q_{n+1}>q_{n}^{2}-q_{n}+1$ for all $n \geq 1$ then $\sum_{n=1}^{\infty} 1 / q_{n}$ is irrational.

This applies to sequences $q_{n}$ that grow doubly exponentially, such as with $q_{n}=2^{2^{n}}$, which leads to the Fredholm number $\sum 2^{-2^{n}}$. Of course, this number doesn't require deep criteria to illuminate its irrationality for it clearly doesn't have a periodic binary expansion, so can't be rational. A less trivial example is if we let $F_{n}$ be the $n$th Fibonacci number and take $q_{n}=F_{2^{n}}$. The required inequality then follows from d'Ocagne's identity. Such fast growth in the denominators might raise fears that any number that satisfies this latter criterion should be a Liouville number, and so in particular transcendental. Indeed, it can be shown by relatively elementary arguments that the Fredholm number is transcendental (see [1, Theorem 13.3.1]). But this isn't the case in general. Taking $q_{n}=F_{2^{n}}$ gives the Millin series, which is in fact

$$
\sum_{n=1}^{\infty} \frac{1}{F_{2^{n}}}=\frac{5-\sqrt{5}}{2}
$$

as shown in Honsberger [5]. So these numbers need not be "very" irrational.

While Theorem 2.1 is indubitably an irrationality criterion for series, it has that property that Brun wanted to avoid, namely it treats the numerators and denominators of the summands separately. We now rephrase Brun's notion of irrationality criteria in the more general setting of first order structures.

Definition 2.3 Let $M \subseteq \mathbb{R}$ and let $\mathcal{M}=(M, \ldots)$ be some first order structure with domain $M$ in the language $L$. An irrationality criterion in $\mathcal{M}$ is a pair $(\varphi, f)$, where $\varphi$ is an $L$-formula in $k$ free variables, $\varphi\left(v_{1}, \ldots, v_{k}\right), f$ is a definable function with domain some subset of $M^{\ell}$ and codomain $M$, and such that the following property holds: for any sequence $\left(a_{n}\right) \in \ell^{1}\left(M \cap \mathbb{Q}^{+}\right)$, if

(1) eventually $\mathcal{M} \models \varphi\left(a_{n+1}, a_{n+2}, \ldots, a_{n+k}\right)$, and

(2) $\lim _{n \rightarrow \infty} f\left(a_{n+1}, a_{n+2}, \ldots, a_{n+\ell}\right)=0$,

then $\sum_{n=1}^{\infty} a_{n}$ is irrational.

Brun's original definition was essentially this where the structure in question was the ordered real numbers expanded by finitely many continuous functions. The limit statement in the above definition may look out of place amidst this discussion of first order statements, but Brun allowed such a limit statement with a continuous function 
and we lose nothing by including it since we can always take the function $f$ to be identically zero, and indeed we will do this for the rest of this section. Note that we can also check several limits with functions, say $f_{1}, \ldots, f_{r}$, by the usual trick of defining $f=f_{1}^{2}+\ldots+f_{r}^{2}$.

In fact there are, trivially, irrationality criteria in any structure $\mathcal{M}$, just take some sentence $\varphi$ such that $\mathcal{M} \not \models \varphi$. Or take a formula that the summands in a convergent series can't eventually satisfy, say $v>1$. Similarly if $M \cap \mathbb{Q}^{+}=\emptyset$ then the definition holds for any formula $\varphi(\vec{x})$. Situations that fall into any of these camps are clearly trivial, so for the rest of this paper they will be ignored.

One less trivial way of abusing the above definition is to cherry pick the domain $M$ so that any mildly controlled sequence of summands gives an irrational sum. For example, if $M=\left\{F_{2^{n}}^{-1}: n \geq 1\right\}$ then any decreasing sequence of terms from $M$ is both absolutely convergent and, by Corollary 2.2, will lead to an irrational sum. So the formula $v_{2}<v_{1}$ gives an irrationality criterion in $(M,<)$.

This is of course cheating. By picking $M$ in this way one is explicitly controlling the numerators in the summands, sidestepping Brun's objection without really addressing the issue. If one takes a less restrictive domain then one might hope to do better.

Unfortunately, even taking a larger domain can lead to issues. Take, for example, $\mathcal{M}=(\mathbb{Q},+, \cdot)$, the rational field. It is well established that the integers are definable in this structure (Robinson [10] gives the earliest definition). Having defined $\mathbb{Z}$ one can use Lagrange's four squares theorem to define the ordering on $\mathbb{Z}$ (or on $\mathbb{Q}$ as a whole, but this won't be needed). And it is possible to define the set of coprime pairs of integers in $\mathbb{Z}$ since $\operatorname{hcf}(m, n)=1$ is equivalent to

$$
\exists a, b \in \mathbb{Z}, a m+b n=1 .
$$

Armed with these and abusing notation slightly we can write down a formula $\varphi\left(v_{1}, v_{2}\right)$ that says the following:

$$
\begin{aligned}
\exists p_{1}, q_{1}, p_{2}, q_{2} \in \mathbb{Z}^{+}, & \\
q_{1} v_{1}=p_{1} \wedge q_{2} v_{2}=p_{2} & \wedge \operatorname{hcf}\left(p_{1}, q_{1}\right)=\operatorname{hcf}\left(p_{2}, q_{2}\right)=1 \\
& \wedge p_{1}\left(q_{2}-1\right)>p_{2} q_{1}\left(q_{1}-1\right) .
\end{aligned}
$$

Given a sequence $\left(a_{n}\right) \in \ell^{1}\left(\mathbb{Q}^{+}\right)$, if eventually $\mathcal{M} \models \varphi\left(a_{n+1}, a_{n+2}\right)$ then all this is saying is that, ignoring finitely many rational terms from the series, we have $a_{n}=p_{n} / q_{n}$ and

$$
\frac{p_{n}}{q_{n}\left(q_{n}-1\right)}>\frac{p_{n+1}}{q_{n+1}-1}
$$


Hence, by Theorem 2.1, $\sum_{n=1}^{\infty} a_{n}$ is irrational.

Again, this is blatant cheating. By allowing $\mathbb{Z}$ to be defined one may as well write the sequence $\left(a_{n}\right)$ as $\left(p_{n} / q_{n}\right)$ and feed the two sequences $p_{n}$ and $q_{n}$ to one's criterion separately. By increasing the domain to $\mathbb{R}$ one can hope to avoid this situation, although clearly there are still numerous structures with domain $\mathbb{R}$ in which the integers are definable. This may be for trivial reasons, like including a predicate for the integers. Or there may be less trivial if not exactly subtle reasons, say the expansion of $\mathbb{R}$ by all analytic functions $f: \mathbb{R} \rightarrow \mathbb{R}$ in which one can then define $\mathbb{Z}=\{x: \sin (\pi x)=0\}$. What would be ideal is a structure where $\mathbb{Z}$ cannot be defined, nor sets like $\left\{F_{2^{n}}^{-1}: n \geq 1\right\}$ that gave trouble earlier, nor in fact any infinite discrete set. Fortunately a large class of such structures have been extensively studied and are well understood.

\section{O-minimal irrationality criteria}

O-minimal structures were introduced in the 1980s to generalise real algebraic geometry (see Pillay and Steinhorn [8, 9] and Knight, Pillay and Steinhorn [6]). They are expansions of the real ordered field with a simple requirement that nonetheless makes them highly congenial to work with.

Definition 3.1 A structure $\mathcal{M}=(\mathbb{R},+, \cdot,<, \ldots)$ is called $o$-minimal if the only definable subsets of $\mathbb{R}$ in this structure are finite unions of points and intervals, where an interval may have endpoints in $\mathbb{R} \cup\{ \pm \infty\}$.

By including the ordering in the definition one is guaranteeing that points and intervals will be definable in $\mathbb{R}$, and so the definition just says that the other functions and relations one has added haven't introduced any new definable subsets in the domain. This doesn't stop new definable sets from appearing in $\mathbb{R}^{n}$ for $n>1$, and indeed there may be lots of new definable sets in these higher dimensions. Nevertheless, this simple condition ensures that the structures are tame to work in, yet interesting enough to warrant the attention. Proofs of all the properties of o-minimal structures that are used below can be found in Lou van den Dries' excellent book [4].

If we use the same definition as before for an irrationality criterion in $\mathcal{M}$ then the presence of the first order formula $\varphi$ corresponds to asking that there be a definable set $X \subseteq \mathbb{R}^{k}$ for some $k$ such that if eventually $\left(a_{n+1}, \ldots, a_{n+k}\right) \in X$ (and the limit condition holds) then $\sum a_{n}$ is irrational. This definition is not actually that useful as will be shown later. Instead we are more faithful to Brun's original definition as follows. 
Definition 3.2 An o-minimal irrationality criterion is a triple consisting of an o-minimal structure $\mathcal{M}$, an open definable set $X \subset \mathbb{R}^{k}$, and a definable function $f:(0, \infty)^{\ell} \rightarrow \mathbb{R}$ that is continuous on the (open) set $U$, with the following properties.

(1) There exists a sequence $\left(a_{n}\right) \in \ell^{1}\left(\mathbb{Q}^{+}\right)$such that eventually $\left(a_{n+1}, \ldots, a_{n+k}\right) \in X$, $\left(a_{n+1}, \ldots, a_{n+\ell}\right) \in U$, and

$$
\lim _{n \rightarrow \infty} f\left(a_{n+1}, \ldots, a_{n+\ell}\right)=0 .
$$

(2) For any sequence $\left(a_{n}\right) \in \ell^{1}\left(\mathbb{Q}^{+}\right)$that satisfies the conditions of property (1), the number $\sum_{n=1}^{\infty} a_{n}$ is irrational.

A sequence $\left(a_{n}\right)$ that satisfies the conditions of property (1) will be said to satisfy $(\mathcal{M}, X, f)$.

It's worth noting that one can take $X=\mathbb{R}$ or $f=0$ to omit either condition in property (1), so including the limit only increases one's options.

Requiring that the summands eventually fall into the open set $U$ upon which $f$ is continuous may seem overly restrictive, but it is in fact necessary. Allowing a closed set $U$ can put us back in the situation of the summands being forced to belong to some distinguished subset, a situation we are trying to rule out. Allowing discontinuous functions is also problematic, since pathological behaviour can hide along the discontinuities, thus negating the generically tame behaviour of o-minimal structures. Examples will be given later to demonstrate these issues, but for now we concentrate on the definition given above.

Since o-minimal structures forbid the skullduggery used earlier to break rational numbers into their numerators and denominators, any o-minimal irrationality criterion should be an honest answer to Brun's question. Moreover, there are many o-minimal structures out there; indeed, there is no limit to them, in the sense of there being no largest structure of which every other o-minimal structure is a reduct (Rolin, Speissesser and Wilkie [11]). It is thus not without the bounds of reason that somewhere in this vast universe of well-behaved structures there exists a set and a function that can detect at least some irrational numbers, if not all of them. But it turns out this is too much to ask.

Theorem 3.3 There are no o-minimal irrationality criteria.

The proof follows the original work of Brun and Knudsen in [3] rather closely. We carry out the proofs in full to satisfy the reader that no properties not enjoyed by o-minimal structures are used. The premise of the proof is to show that if $(\mathcal{M}, X, f)$ is a triple 
as in the definition and if $\left(a_{n}\right) \in \ell^{1}\left(\mathbb{Q}^{+}\right)$satisfies this triple, and if moreover $\sum a_{n}$ is irrational then one can construct a sequence $\left(b_{n}\right) \in \ell^{1}\left(\mathbb{Q}^{+}\right)$such that $\left(b_{n}\right)$ satisfies $(\mathcal{M}, X, f)$ but where $\sum b_{n}$ is rational. Hence any putative o-minimal irrationality criterion that makes it as far as property (1) in the definition will fall at property (2).

First we will show that if $\left(a_{n}\right)$ satisfies $(\mathcal{M}, X, 0)$ then so do sequences sufficiently close to $\left(a_{n}\right)$. This is a simple consequence of $X$ being open.

Lemma 3.4 Let $X \subset(0,1)^{k}$ be an open set that is definable in the o-minimal structure $\mathcal{M}$ and let $\left(a_{n}\right) \in \ell^{1}\left(\mathbb{R}^{+}\right)$. If eventually

$$
\left(a_{n+1}, \ldots, a_{n+k}\right) \in X
$$

then there is a sequence $\left(\varepsilon_{n}\right) \in \ell^{1}\left(\mathbb{R}^{+}\right)$with the property that for any sequence of real numbers $\left(\delta_{n}\right)$ with $\left|\delta_{n}\right| \leq \varepsilon_{n}$, eventually

$$
\left(a_{n+1}+\delta_{n+1}, \ldots, a_{n+k}+\delta_{n+k}\right) \in X .
$$

Proof Let $N>k$ be a natural number for which $\left(a_{n+1}, \ldots, a_{n+k}\right) \in X$ whenever $n>N$. Set $\bar{\varepsilon}_{n}=1$ for $1 \leq n \leq N$. Since $X$ is open, for each $n>N$ there is a closed cube around the point $\left(a_{n+1}, \ldots, a_{n+k}\right)$ that is contained in $X$, say

$$
B_{n+1}=\left\{\left(b_{1}, \ldots, b_{k}\right) \in \mathbb{R}^{k}:\left|a_{n+i}-b_{i}\right| \leq w_{n+1}, 1 \leq i \leq k\right\} \subset X
$$

with $w_{n}>0$ for all $n$. If we set $\bar{\varepsilon}_{n}=\min \left(w_{n}, w_{n-1}, \ldots, w_{n-k+1}, 1\right)$ for $n>N$ and then let $\varepsilon_{n}=2^{-n} \bar{\varepsilon}_{n}$ then clearly $\left(\varepsilon_{n}\right) \in \ell^{1}\left(\mathbb{R}^{+}\right)$and moreover if $\left|\delta_{n}\right| \leq \varepsilon_{n}$ then for $n>N$ we have

$$
\left(a_{n+1}+\delta_{n+1}, \ldots, a_{n+k}+\delta_{n+k}\right) \in B_{n+1} \subset X
$$

as required.

The next stage is to show the same kind of result when $\left(a_{n}\right)$ satisfies $(\mathcal{M}, \mathbb{R}, f)$. Note that while definable functions are mostly continuous, they can be discontinuous on a lower-dimensional subset of their domain. As alluded to above, the continuity condition in the lemma below (and so in the definition above) prevent one finding a sequence $\left(a_{n}\right)$ that will ride along such a discontinuity, while any sequence near $\left(a_{n}\right)$, but not on the discontinuous part of the function's domain, will have, essentially, nothing to do with $\left(a_{n}\right)$. This is not such a big restriction on $f$ since definable functions will, generically, be continuous near the origin. In the following lemma we restrict $f$ to the open subset of its domain where it is continuous since the discontinuous part is not involved anyway. 
Lemma 3.5 Let $f: U \rightarrow \mathbb{R}$ be a definable continuous function in $\mathcal{M}$ with $U \subset(0, \infty)^{\ell}$ an open set. Let $\left(a_{n}\right) \in \ell^{1}\left(\mathbb{R}^{+}\right)$be such that eventually $\left(a_{n+1}, \ldots, a_{n+\ell}\right) \in U$ and

$$
\lim _{n \rightarrow \infty} f\left(a_{n+1}, \ldots, a_{n+\ell}\right)=0 .
$$

Then there is a sequence $\left(\varepsilon_{n}\right) \in \ell^{1}\left(\mathbb{R}^{+}\right)$with the property that for any sequence of real numbers $\left(\delta_{n}\right)$ with $\left|\delta_{n}\right| \leq \varepsilon_{n}$, we eventually have $\left(a_{n+1}+\delta_{n+1}, \ldots, a_{n+\ell}+\delta_{n+\ell}\right) \in U$ and

$$
\lim _{n \rightarrow \infty} f\left(a_{n+1}+\delta_{n+1}, \ldots, a_{n+\ell}+\delta_{n+\ell}\right)=0
$$

Proof Let $N>\ell$ be such that $\left(a_{n+1}, \ldots, a_{n+\ell}\right) \in U$ for $n \geq N$. Since $f$ is continuous in the open set $U$, around each of the points $\left(a_{n+1}, \ldots, a_{n+\ell}\right)$ there is an open neighbourhood $V_{n+1} \subset U$ such that for any $\left(v_{n+1}, \ldots, v_{n+\ell}\right) \in V_{n+1}$ we have

$$
\left|f\left(a_{n+1}, \ldots, a_{n+\ell}\right)-f\left(v_{n+1}, \ldots, v_{n+\ell}\right)\right|<2^{-n} .
$$

Within each open set $V_{n+1}$ there is of course a closed cube $B_{n+1}$, say

$$
B_{n+1}=\left\{\left(b_{1}, \ldots, b_{\ell}\right) \in \mathbb{R}^{\ell}:\left|a_{n+i}-b_{i}\right| \leq w_{n+1}, 1 \leq i \leq \ell\right\} \subset V_{n+1}
$$

with all $w_{n}>0$. For convenience we let $w_{i}=1$ for $i<N$. Now let $\bar{\varepsilon}_{n}=$ $\min \left(w_{n}, w_{n-1}, \ldots, w_{n-\ell+1}, 1\right)$, and finally set $\varepsilon_{n}=2^{-n} \bar{\varepsilon}_{n}$. Clearly $\left(\varepsilon_{n}\right) \in \ell^{1}\left(\mathbb{R}^{+}\right)$. Now if $\left|\delta_{n}\right| \leq \varepsilon_{n}$ for all $n$ then we eventually have

$$
\left(a_{n+1}+\delta_{n+1}, \ldots, a_{n+\ell}+\delta_{n+\ell}\right) \in V_{n+1} \subset U
$$

and

$$
\left|f\left(a_{n+1}+\delta_{n+1}, \ldots, a_{n+\ell}+\delta_{n+\ell}\right)\right| \leq\left|f\left(a_{n+1}, \ldots, a_{n+\ell}\right)\right|+2^{-n} .
$$

Taking the limit as $n \rightarrow \infty$ proffers the result.

These last two lemmas are combined in the obvious way.

Corollary 3.6 Let $\mathcal{M}$ be an o-minimal structure, $X \subset \mathbb{R}^{k}$ be an open set that is definable in $\mathcal{M}$, and $f: U \rightarrow \mathbb{R}$ be a continuous definable function with $U \subset(0, \infty)^{\ell}$ an open set. Suppose that $\left(a_{n}\right) \in \ell^{1}\left(\mathbb{R}^{+}\right)$is a sequence such that eventually $\left(a_{n+1}, \ldots, a_{n+k}\right) \in X$, $\left(a_{n+1}, \ldots, a_{n+\ell}\right) \in U$, and

$$
\lim _{n \rightarrow \infty} f\left(a_{n+1}, \ldots, a_{n+\ell}\right)=0 .
$$

Then there is a sequence $\left(\varepsilon_{n}\right) \in \ell^{1}\left(\mathbb{R}^{+}\right)$with the property that for any sequence of real numbers $\left(\delta_{n}\right)$ with $\left|\delta_{n}\right| \leq \varepsilon_{n}$, we eventually have $\left(a_{n+1}+\delta_{n+1}, \ldots, a_{n+k}+\delta_{n+k}\right) \in X$, $\left(a_{n+1}+\delta_{n+1}, \ldots, a_{n+\ell}+\delta_{n+\ell}\right) \in U$ and

$$
\lim _{n \rightarrow \infty} f\left(a_{n+1}+\delta_{n+1}, \ldots, a_{n+\ell}+\delta_{n+\ell}\right)=0 .
$$


So if we have a sequence $\left(a_{n}\right) \in \ell^{1}\left(\mathbb{R}^{+}\right)$that satisfies the triple $(\mathcal{M}, X, f)$ then we can perturb the sequence slightly term-by-term to get a new sequence that also satisfies the triple. It remains to show that arbitrarily close to any rational sequence $\left(a_{n}\right)$ with $\sum a_{n}$ irrational, there is a rational sequence $\left(b_{n}\right)$ with $\sum b_{n}$ rational. This is not difficult to achieve using telescoping sums, as in Brun and Knudsen [3]. We use a slightly different form of their argument below.

Lemma 3.7 Let $\left(\varepsilon_{n}\right) \in \ell^{1}\left(\mathbb{R}^{+}\right)$with $\sum \varepsilon_{n}=\varepsilon$. Then for any $\gamma \in[0, \varepsilon)$ there is a sequence $\left(\gamma_{n}\right) \in \ell^{1}(\mathbb{Q})$ with, eventually, $\gamma_{n} \in\left[0, \varepsilon_{n}\right)$ and with $\sum \gamma_{n}=\gamma$.

This suffices for our purpose, since if $\sum a_{n}=a$ is irrational and $\left(\varepsilon_{n}\right)$ is the sequence given by Corollary 3.6 and $\sum \varepsilon_{n}=\varepsilon$ then we can take some rational number $b$ with $a<b<a+\varepsilon$ and find a rational sequence $\left(\gamma_{n}\right)$ with $\gamma_{n} \in\left[0, \varepsilon_{n}\right)$ for large $n$ and with $\sum \gamma_{n}=b-a<\varepsilon$. Then $\left(a_{n}+\gamma_{n}\right)$ is a rational sequence with $\sum\left(a_{n}+\gamma_{n}\right)=a+b-a=b \in \mathbb{Q}$.

Proof The "eventually" in the statement of the lemma allows the first $N$ terms of the sequence $\left(\gamma_{n}\right)$ to be picked however we want without worrying about the sequence $\left(\varepsilon_{n}\right)$. In fact we only need to pick $\gamma_{1}$ this way, the rest of the sequence will satisfy the other conditions.

If $\gamma$ is rational then just take $\gamma_{1}=\gamma$ and $\gamma_{n}=0$ for $n \geq 2$. This clearly satisfies the conclusion of the lemma.

Now suppose that $\gamma$ is irrational and let the continued fraction expansion of $\gamma$ be

$$
\gamma=\left[c_{0} ; c_{1}, c_{2}, \ldots\right] .
$$

Let $p_{n} / q_{n}$ be the $2 n$th convergent to $\gamma$, that is

$$
\frac{p_{n}}{q_{n}}=\left[c_{0} ; c_{1}, c_{2}, \ldots, c_{2 n}\right]
$$

These even convergents $p_{n} / q_{n}$ form an increasing sequence of rational numbers that converges to $\gamma$. Using the standard properties of the convergents we have

$$
0<\alpha-\frac{p_{n}}{q_{n}}<\frac{1}{c_{2 n+1} q_{n}^{2}} \leq \frac{1}{q_{n}^{2}} .
$$

So in particular for any $n<m$ we have

$$
0<\frac{p_{m}}{q_{m}}-\frac{p_{n}}{q_{n}}<\frac{1}{q_{n}^{2}}
$$

Journal of Logic \& Analysis 7:1 (2015) 
Given the sequence $\left(\varepsilon_{n}\right)$ we thus pick an increasing sequence of positive integers $r(k)$ such that for all $k$

$$
\frac{1}{q_{r(k)}^{2}} \leq \varepsilon_{k}
$$

so that if we let $\gamma_{1}=p_{r(1)} / q_{r(1)}$ and for $n>1$ let

$$
\gamma_{n}=\frac{p_{r(n+1)}}{q_{r(n+1)}}-\frac{p_{r(n)}}{q_{r(n)}}
$$

then for $n \geq 2$ we have $0<\gamma_{n}<\varepsilon_{n}$ and

$$
\sum_{k=1}^{n} \gamma_{k}=\frac{p_{r(n+1)}}{q_{r(n+1)}} \rightarrow \gamma
$$

This proves Theorem 3.3. However, we should properly address the aforementioned issues with the definition given of an o-minimal irrationality criterion.

Two of the lesser issues are to do with the function $f$. Including the limit may be a point of contention, but as mentioned earlier one can always ignore it, so including it only rules out a wider class of possible criteria. A less clear cut issue is the way that the discontinuities of $f$ are dealt with, namely ignoring them altogether. We will give an example at the end of the paper as to why this is necessary, but it is also not such a large issue due to the well-behaved nature of definable functions.

The third and final issue is the use of only open definable sets $X$. This renders our Lemma 3.4 into an exercise in remembering the definition of "open". One might hope that o-minimal structures allow one to relax this condition. But as we said earlier this is not the case. The simplest case of non-open sets are just graphs of functions, and we will see below that even in the one-dimensional case these are problematic. By cell decomposition, all definable sets are the union of finitely many open sets and graphs of various dimensions. By the above if a definable set contains an open piece near the origin so that a rational sequence can stay in the set and have a convergent series, then the set can't belong to an irrationality criterion. This really leaves the situation where the set is the graph of a function that gets close to the origin. In the lowest dimensional case we can get the following result that is partly analogous to the above lemmas.

Lemma 3.8 Let $f: \mathbb{R}^{+} \rightarrow \mathbb{R}^{+}$be a definable function in an o-minimal structure $\mathcal{M}$. Suppose $\left(a_{n}\right) \in \ell^{1}\left(\mathbb{R}^{+}\right)$satisfies $a_{n+1}=f\left(a_{n}\right)$ for $n \geq 1$. Suppose further that $\sum a_{n}$ is irrational. Then for any $\varepsilon>0$ there is a sequence $\left(b_{n}\right) \in \ell^{1}\left(\mathbb{R}^{+}\right)$with $\left|a_{1}-b_{1}\right|<\varepsilon$ such that $b_{n+1}=f\left(b_{n}\right)$ for all $n \geq 1$ and with $\sum b_{n}$ rational. 
Proof Let $f_{1}(x)=x$ and for $n \geq 1$ let $f_{n+1}(x)=f \circ f_{n}(x)$. Observe that $a_{n}=f_{n}\left(a_{1}\right)$.

First we claim that for some suitably small $\eta>0, f^{\prime}$ exists on $(0, \eta)$ and moreover $0<f^{\prime}(x)<1$ for all $x \in(0, \eta)$. Indeed, by cell decomposition there is $\eta_{1}$ such that $f^{\prime}$ exists on $\left(0, \eta_{1}\right)$, and since the set $\left\{x: f^{\prime}(x)=0\right\}$ is definable there is $\eta_{2} \in\left(0, \eta_{1}\right)$ such that on $\left(0, \eta_{2}\right)$ either $f^{\prime}<0$ or $f^{\prime}=0$ or $f^{\prime}>0$.

If $f^{\prime}=0$ then $f$ is constant on $\left(0, \eta_{2}\right)$. Since $a_{n} \rightarrow 0$, eventually $a_{n}<\eta_{2}$ and thenceforth $a_{n+1}=f\left(a_{n}\right)=a_{n}$. But this contradicts $a_{n}$ tending to zero.

If $f^{\prime}<0$ then since $\lim _{x \rightarrow 0^{+}} f(x)=0$ we have by the mean value theorem that, for large enough $n$,

$$
\frac{f\left(a_{n}\right)}{a_{n}}=f^{\prime}\left(\xi_{n}\right)<0
$$

for some $\xi_{n}<\eta_{2}$. So either $a_{n}<0$ or $f\left(a_{n}\right)<0$. But by definition $a_{n}>0$ and $f\left(a_{n}\right)=a_{n+1}>0$, so this can't happen.

This establishes that $f^{\prime}>0$ on $\left(0, \eta_{2}\right)$. The set $\left\{x: f^{\prime}(x) \geq 1\right\}$ is definable, so there must be $\eta \leq \eta_{2}$ such that on $(0, \eta)$ either $f^{\prime}<1$ or $f^{\prime} \geq 1$. Suppose the latter holds, then by the mean value theorem for all large $n$ there is $\xi_{n}^{\prime} \in(0, \eta)$ with

$$
\frac{f\left(a_{n}\right)}{a_{n}}=f^{\prime}\left(\xi_{n}^{\prime}\right) \geq 1 \text {. }
$$

In other words, for all large $n$ we have $a_{n+1} \geq a_{n}$. But $\left(a_{n}\right)$ is a positive sequence that tends to zero, so this can't happen.

This gives an interval $(0, \eta)$ on which $0<f^{\prime}(x)<1$, hence on this interval we have that if $x<y$ then $f(x)<f(y)$, and so by induction if $x<y$ then $f_{n}(x)<f_{n}(y)$ for any $n \geq 0$. Without loss of generality we may assume the sequence $\left(a_{n}\right)$ lies only in this interval. In particular, for any $x \in\left(0, a_{1}\right)$ we have $f_{n}(x)<f_{n}\left(a_{1}\right)=a_{n}$. But by hypothesis $\sum a_{n}$ converges, so by the Weierstrass M-test the function $F(x)=\sum_{n=1}^{\infty} f_{n}(x)$ converges uniformly to a continuous function on $\left(0, a_{1}\right)$. Note that this resulting function is not constant due to the aforementioned fact that $f_{n}(x)<f_{n}(y)$ whenever $x<y<a_{1}$. So in particular for any $\varepsilon>0$ there exists $b_{1} \in\left(a_{1}-\varepsilon, a_{1}\right)$ for which $F\left(b_{1}\right)$ is rational. But $F\left(b_{1}\right)=\sum f_{n}\left(b_{1}\right)$ so setting $b_{n}=f_{n}\left(b_{1}\right)$ gives the required sequence.

The reason this result is only a partial analogue to the preceding lemmas is that the resulting sequence $\left(b_{n}\right)$ is only guaranteed to have real terms, not rational ones. The problem is that we only really get to pick one term in the sequence: $b_{1}$. The rest of the terms are determined by the function $f$. There are very simple functions $f$ for which the function $F$ as constructed above returns irrational values for any rational argument. 
Indeed, Mahler proved in [7] that if one takes $f(x)=x^{2}$ and constructs $F$ as above, then for any rational $x \in(0,1)$ the value $F(x)$ is transcendental, that is

$$
\sum_{n=0}^{\infty}\left(\frac{p}{q}\right)^{2^{n}}
$$

is transcendental for any natural numbers $p<q$.

This example of Mahler also reveals why, when looking at o-minimal irrationality criteria $(\mathcal{M}, X, f)$, we needed to take pains to avoid the discontinuous part of $f$. Consider, say, the function $f:(0,1)^{2} \rightarrow \mathbb{R}$ given by

$$
f\left(x_{1}, x_{2}\right)= \begin{cases}x_{2}^{2} & \text { if } x_{2}=x_{1}^{2} \\ 1 & \text { otherwise }\end{cases}
$$

For almost any sequence $\left(a_{n}\right) \in \ell^{1}\left(\mathbb{Q}^{+}\right)$, we will have $\lim f\left(a_{n}, a_{n+1}\right)=1$ and so the sequence won't satisfy $(\mathcal{M}, \mathbb{R}, f)$. However, if $a_{n}$ satisfies $a_{n+1}=a_{n}^{2}$ for all large $n$ then

$$
\lim _{n \rightarrow \infty} f\left(a_{n}, a_{n+1}\right)=\lim _{n \rightarrow \infty} a_{n+1}^{2}=0 .
$$

But these are precisely the sequences that Mahler proved always summed to transcendental numbers. Hence if we include such a function $f$ and don't demand that sequences stay away from their discontinuous part then $(\mathcal{M}, \mathbb{R}, f)$ fulfills all the conditions to be an o-minimal irrationality criteria. We discount these since, as evinced above, they are really descriptions of functions that map $\mathbb{Q}$ into the irrational numbers rather than being an actual criterion we can check arbitrary series against.

\section{Acknowledgements}

Thanks to the organisers of the Applications of O-Minimality to Analysis and Number Theory workshop in Passau in September 2013, where this research was first aired in public. Thanks also to the referee for several useful comments. The author was supported by the Heilbronn Institute for Mathematical Research.

\section{References}

[1] J-P Allouche, J Shallit, Automatic sequences: theory, applications, generalizations, Cambridge University Press (2003)

[2] V Brun, Ein Satz über Irrationalität, Archiv for Mathematik og Naturvidenskab (Kristiania) 31 (1910) 6 
[3] V Brun, F F Knudsen, On the Possibility of Finding Certain Criteria for the Irrationality of a Number Defined as a Limit of a Sequence of Rational Numbers, Mathematica Scandinavica 31 (1972) 231-236

[4] L van den Dries, Tame Topology and O-minimal structures, Cambridge University Press (1998)

[5] R Honsberger, Mathematical Gems III, volume 9 of Dolciani Mathematical Expositions, The Mathematical Association of America (1985)

[6] J Knight, A Pillay, C Steinhorn, Definable sets in ordered structures II, Transactions of the American Mathematical Society 295 (1986) 593-605

[7] K Mahler, Arithmetische Eigenschaften der Lösungen einer Klasse von Funktionalgleichungen, Mathematische Annalen 101 (1929) 342-366

[8] A Pillay, C Steinhorn, Definable sets in ordered structures I, Transactions of the American Mathematical Society 295 (1986) 565-592

[9] A Pillay, C Steinhorn, Definable sets in ordered structures III, Transactions of the American Mathematical Society 309 (1988) 469-476

[10] J Robinson, Definability and decision problems in arithmetic, The Journal of Symbolic Logic 14 (1949) 98-114

[11] J-P Rolin, P Speissegger, A J Wilkie, Quasianalytic Denjoy-Carleman classes and o-minimality, J. Amer. Math. Soc. 16 (2003) 751-777

Heibronn Institute for Mathematical Research, Howard House, Queen's Avenue, Bristol, BS8 $1 S D, U K$

lee.butler@bristol.ac.uk

Received: 19 August $2014 \quad$ Revised: 13 February 2015 